\title{
Investigation into the value of Papanicolaou stained cervical smears for the diagnosis of chlamydial cervical infection
}

\author{
GE FORSTER,${ }^{*}$ I COOKEY,${ }^{*}$ PE MUNDAY,${ }^{*} \ddagger$ PI RICHMAN,$\dagger \S$ R JHA,$\dagger$ D COLEMAN,$\dagger$ \\ BJ THOMAS, $\ddagger$ DA HAWKINS,${ }^{*} \ddagger$ RT EVANS, $\ddagger$ D TAYLOR-ROBINSON $\ddagger$
}

From the * Departments of Genito-urinary Medicine and †Pathology, St Mary's Hospital and Medical School, London W2, and the $\ddagger$ Division of Sexually Transmitted Diseases, MRC Clinical Research Centre, Harrow, Middlesex.

SUMMARY Forty five (37\%) of 121 female contacts of men with non-gonococcal urethritis or gonorrhoea were chlamydia positive, as judged by isolation or by detecting elementary bodies in smears with a fluorescein labelled chlamydial monoclonal antibody. Only six (13\%) of these, however, had Papanicolaou stained smears in which there were inclusion like changes suggestive of chlamydial infection. Furthermore, of 15 patients who had such cytological changes, chlamydiae were detected in only six and the abnormalities were found also in Papanicolaou stained smears from $10(13 \%)$ of the 76 chlamydia negative patients. Modifying the Papanicolaou stained smears by including endocervical material did not increase sensitivity. In addition, destaining and restaining them with the monoclonal antibody was time consuming and the results were unreliable. The staining of cervical smears with Papanicolaou reagent is a technique of low sensitivity and specificity for diagnosing or screening for chlamydial cervical infection and cannot be recommended.

There are no characteristic clinical features of infection with Chlamydia trachomatis in women, although a presumptive diagnosis of chlamydial infection may be made in women who have a history of sexual contact with men with non-gonococcal urethritis or gonorrhoea.' A definitive diagnosis requires the isolation of chlamydiae in cell culture or the use of a monoclonal antibody to detect chlamydial elementary bodies. The former procedure is time consuming, expensive, and not always available routinely, ${ }^{2}$ and the latter procedure is expensive and not yet used widely.

Many women undergo cytological screening at intervals during their reproductive lives to detect premalignant cervical changes. The possibility of detecting chlamydial infection simultaneously in these Papanicolaou stained (PAP) smears is attractive, but the sensitivity and specificity of this method have been the subject of some controversy. Naib studied PAP smears from newborn infants with con-

Accepted for publication 10 December 1984

§Present address: Imperial Cancer Research Fund, Lincoln's Inn Fields, London WC2A 3PX. junctivitis and from the cervix of their mothers and proposed criteria for identifying cytoplasmic inclusions which he regarded as being suggestive of chlamydial infection. These criteria were modified later by Gupta et al, ${ }^{4}$ who examined inclusions in PAP cervical smears from 160 women. In both studies the diagnosis of chlamydial infection in most patients was not confirmed by culture or by other specific procedures. Despite reports from several groups of workers ${ }^{5-8}$ that routine cytology is not a reliable diagnostic technique for detecting chlamydial infection, it is not without enthusiastic support. ${ }^{y}$ In an earlier study ${ }^{10}$ we found some false positive PAP smears: in other words, smears considered to contain chlamydial inclusions that were not confirmed as chlamydia positive by the isolation technique for $C$ trachomatis. This may have been due to various factors which could have affected the sensitivity of the isolation procedure. The present study was therefore designed to reassess the sensitivity and specificity of examining PAP smears to diagnose chlamydial infection. In part of the study the procedure for taking PAP smears was modified to enhance the chance of detecting inclusions con- 


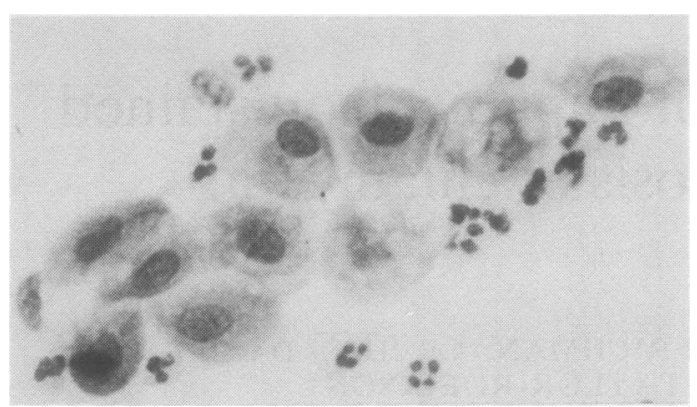

Fig. 1 Cervical smear. Squamous epithelial cells in which there is cytoplasmic granularity and vacuolar inclusions. Papanicolaou $\times 400$.

sidered to be specific for chlamydial infection. Furthermore, in order to clarify any discrepancies between the results of examining PAP smears and culture for chlamydiae, an additional specimen was taken and stained by a fluorescein labelled monoclonal antibody against $C$ trachomatis, a technique which is highly specific and sensitive."

\section{Patients and methods}

\section{STUDY POPULATION}

The study population consisted of women attending the Praed Street Clinic. All were known sexual contacts of men with non-gonococcal urethritis or gonorrhoea. Women who were menstruating, those who had taken antibiotics within the previous month, and those who were pregnant were excluded.

\section{PAPANICOLAOU STAINED SMEARS}

In the first group of patients (A) a wooden Ayre's spatula was used to take cervical material in the conventional manner. This was spread on a glass slide and fixed immediately in $95 \%$ ethanol. In the second group of patients (B) additional material was obtained from the endocervical canal using a cotton tipped swap (MW108, Medical Wire and Equipment $\mathrm{Co}$ ). This was smeared on the same slide as that used for the conventional PAP smear. Staining was by the standard Papanicolaou technique, and

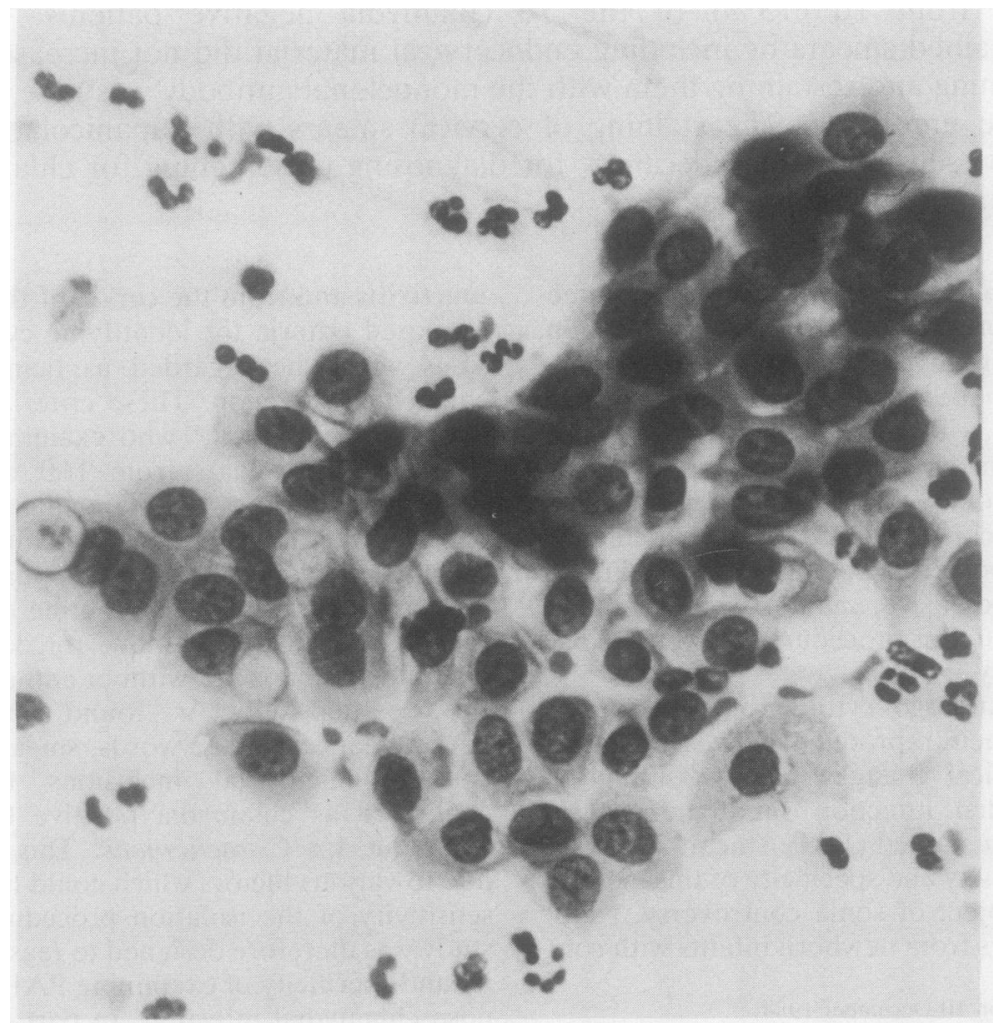

Fig. 2 Cervical smear. The cells at the left of this group of squamous metaplastic cells are vacuolated and contain inclusion bodies suggestive of chlamydial infection. Papanicolaou $\times 400$. 
the slides were viewed by three independent observers (PIR, RJ, and DC) without knowledge of the chlamydial culture results. The minimum criterion for the diagnosis of chlamydial infection was the presence of a finely granular cytoplasm, in endocervical or metaplastic cells, in which single or multiple inclusions could be seen (Figs. 1 and 2). The observers initially spent up to $30 \mathrm{~min}$ examining each slide, but with experience this was reduced to $10 \mathrm{~min}$.

\section{SPECIMENS FOR DIRECT}

IMMUNOFLUORESCENCE TESTS

Specimens to be stained with the chlamydial monoclonal antibody were obtained by rotating a cotton tipped swab, as above, within the endocervical canal. In the first group of patients (A) the swab was smeared on a glass slide as for a PAP smear. In the second group (B) it was rubbed over a more confined area on a prepared standard slide. The smears were air dried and fixed with acetone before being stored at $4^{\circ} \mathrm{C}$. They were stained using a commercially available chlamydial monoclonal antibody (Microtrak, Syva UK) according to the manufacturer's instructions. The recognition of one or more fluorescing bodies of characteristic shape and size was regarded as indicative of chlamydial infection. ${ }^{11}$

\section{SPECIMENS FOR CHLAMYDIAL ISOLATION}

An endocervical specimen was taken with a polyester sponge swab, which was expressed in sucrosephosphate transport medium (2SP) containing $10 \%$ heat inactivated fetal calf serum. Then the medium was snap frozen in liquid nitrogen for transport to and storage in the laboratory. Cycloheximide treated McCoy cells were used to isolate $C$ trachomatis. They were stained with Giemsa reagent and examined by dark ground microscopy.12 These specimens were taken before routine tests to exclude other genital tract infections.

\section{Results}

Cervical smears for Papanicolaou staining and for

Table 1 Relation between the results of seeking inclusions in Papanicolaou stained (PAP) smears and those of definitive detection methods for cervical chlamydial infection in patients in group $A$

\begin{tabular}{lc}
\hline Test result for & No of patients \\
\hline Chlamydia positive patients & \\
PAP positive & 5 \\
PAP negative & 14 \\
Chlamydia negative patients & 1 \\
PAP positive & 27 \\
PAP negative & 1 \\
PAP equivocal & \\
\hline
\end{tabular}

Table 2 Relation between the results of seeking inclusions in Papanicolaou stained smears and those of definitive detection methods for cervical chlamydial infection in patients in group $B$

\begin{tabular}{lc}
\hline Test result for & No of patients \\
\hline Chlamydia positive patients & 1 \\
PAP positive & 25 \\
PAP negative & 8 \\
Chlamydia negative patients & 39 \\
PAP positive & \\
PAP negative & \\
\hline
\end{tabular}

monoclonal antibody staining and cervical specimens for chlamydial culture were obtained from 121 women. Chlamydiae were detected either by isolation or by monoclonal antibody staining techniques in $45(37 \%)$ of these.

There were 48 patients in group $\mathrm{A}$, who had smears taken in the conventional way for Papanicolaou staining (Table 1). Of 19 patients who were chlamydia positive, only five $(26 \%)$ had inclusions in PAP smears which were suggestive of chlamydial infection. Of the remaining 29 women who were chlamydia negative by both culture and monoclonal antibody staining smear tests, there was one whose PAP smear was recorded as positive and one with an equivocal PAP smear result.

There were 73 patients in group B (Table 2), whose conventional PAP smears were supplemented with smears from the endocervix. This, however, did not afford any advantage. Thus, of 26 patients who were chlamydia positive, only one (4\%) had inclusions suggestive of chlamydial infection seen in the PAP smear. Of the remaining 47 women who were chlamydia negative, there were eight $(17 \%)$ whose PAP smears were recorded as positive.

An attempt was made to show the presence of extracellular chlamydial elementary bodies in PAP smears which had been destained. Eleven destained PAP smears from patients in group B were then restained using the monoclonal antibody staining technique and viewed by a single observer (BT) without knowledge of the earlier results. Six patients were originally chlamydia positive, according to both culture and monoclonal antibody staining techniques, but only three $(50 \%)$ had restained smears which were considered positive. Of the other five who were originally chlamydia negative, one had a restained smear which was considered positive. The restaining procedure was, therefore, insufficiently sensitive or specific probably because it produced brightly fluorescing cells in the background, against which it was difficult to distinguish chlamydial elementary bodies. 


\section{Discussion}

The diagnosis of chlamydial cervical infection using a routinely available and simple test, the PAP smear, would be cost effective and permit screening of low risk populations if it were sensitive and specific. Use of cytology as a screening technique to select patients to be examined for chlamydiae by culture or by a monoclonal antibody staining technique might also be of value ${ }^{7.3}$ if it were sufficiently sensitive.

Cytological changes in PAP smears suggestive of infection by $C$ trachomatis have been reported, ${ }^{34}$ but verification by culture was rarely. sought. In those studies in which culture for chlamydiae has been incorporated, however, the possibility that false negative culture results could have arisen has been an issue. In this study the chlamydial monoclonal antibody was used to support the culture tests. Of 45 patients who were chlamydia positive by chlamydial culture or monoclonal antibody staining smear tests, or both, only six $(13 \%)$ had inclusions in PAP smears which were suggestive of chlamydial infection. This indicated the low sensitivity of the latter technique.

Ten chlamydia negative patients had PAP smears which were reported to contain inclusions consistent with chlamydial infection: the "inclusions" were probably aggregates of bacteria, cell debris, or other artefacts. The diagnosis of chlamydial infection using fluorescein labelled monoclonal antibody to stain smears is based on the detection of extracellular chlamydial elementary bodies. Epithelial cells with cytoplasmic chlamydial inclusions which are intact are seen in less than $5 \%$ of specimens ${ }^{14}$ (and Thomas BJ, unpublished observations), so that it is not surprising that inclusions characteristic of chlamydial infection are rarely seen in PAP smears. It is probable that they are disrupted while taking the specimens to prepare the smears or while making the smears on the glass slides before fixing.

Since chlamydiae infect endocervical or metaplastic cells, the standard technique for PAP smears was modified to include endocervical material. This, however, did not improve the sensitivity of the test. Furthermore, modifying the PAP smear procedure by destaining and then restaining with the fluorescein labelled monoclonal antibody was time consuming and insufficiently sensitive or specific to be used routinely. The results of our study support and extend those of other workers in the contention that the PAP smear is a test of low sensitivity and specificity for detecting chlamydial cervical infection and should not be used for this purpose.

We thank Dr JRW Harris and Dr D Goldmeier for allowing us to examine patients under their care. We are grateful also to the nursing staff of the Praed Street Clinic for their assistance.

\section{References}

Munday PE. Chlamydia infections in women. In: Studd J, ed. Progress in obstetrics and gynaecology, Vol 3, London: Churchill Livingstone, 1983:231-45.

${ }^{2}$ Mârdh P-A, Weström L, Colleen S, Wølner-Hanssen P. Sampling specimen handling, and isolation techniques in the diagnosis of chlamydial and other genital infections. Sex Transm Dis 1981;8:280-5.

' Naib ZM. Cytology of TRIC agent infection of the eye of newborn infants and their mothers genital tracts. Acta Cytol 1970;14:390-5.

4 Gupta PK, Lee EF, Erozan YS, Frost JK, Geddes ST, Donovan PA. Cytologic investigations in chlamydia infection. Acta Cytol 1979; 23:315-20.

s Paavonen J, Purola E. Cytologic findings in cervical chlamydial infection. Med Biol 1980;58:174-8.

- Purola E, Paavonen J. Routine cytology as a diagnostic aid in chlamydial cervicitis. Scand J Infect Dis (suppl) 1982;32:55-8.

' Dorman SA, Danos LM, Wilson DJ, et al. Detection of chlamydial cervicitis by Papanicolaou stained smears and culture. Am J Clin Pathol 1983;79:421-5.

* Giampaolo C, Murphy J, Benes S, McCormack WM. How sensitive is the Papanicolaou smear in the diagnosis of infections with Chlamydia trachomatis? Am J Clin Pathol 1983;80:844 9.

De Borges RJ, Carmona O, Machado H, Esparza J. Chlamydial infection in Papanicolaou-stained cervical smears. Acta Cytol 1984;28:471-6.

${ }^{10}$ Forster G, Jha R, Cheetham D, Munday P, Coleman D, Taylor-Robinson D. Cytological diagnosis of chlamydial infection of female genital tract. Lancet 1983;ii:578-9.

"Thomas BJ, Evans RT, Hawkins DA, Taylor-Robinson D. The sensitivity of detecting Chlamydia trachomatis elementary bodies in smears by use of a fluorescein-labelled monoclonal antibody compared to that of conventional chlamydial isolation. J Clin Pathol 1984;37:812-6.

12 Thomas BJ, Evans RT, Hutchinson GR, Taylor-Robinson D. Early detection of chlamydial inclusions combining the use of cycloheximide-treated McCoy cells and immunofluorescence staining. J Clin Microbiol 1977;6:285-92.

"Medley G. Cytological diagnosis of chlamydial infection of female genital tract. Lancet 1983; 1449.

${ }^{14}$ Tam MR, Stamm WE, Handsfield HH, et al. Cultureindependent diagnosis of Chlamydia trachomatis using monoclonal antibodies. $N$ Engl J Med 1984;310:1146-50.

Requests for reprints to: Dr GE Forster, The Praed Street Clinic, St Mary's Hospital, London W2 IP6, England. 